\title{
Management of pigment epithelium detachment using Spectral Domain Optical Coherence Tomography.
}

\author{
Rebecca Rolf*, Florence Coscas, Gabriel Coscas, Salomon Yves Cohen, Eric H Souied \\ Department of Ophthalmology, University Paris Est Créteil, Centre Hospitalier Intercommunal de Créteil, Créteil, \\ France
}

\begin{abstract}
Pigment epithelium detachment (PED) is a common sign in optical coherence tomography (OCT), but with lots of various characteristics in term of description, aetiology and treatment. Some PED could be asymptomatic, other with metamorphopsia, photopsia, vision loss. The authors describe a short case report about PED but more general a discussion about semiology of the description in OCT to guide the specialist to the correct diagnostic.
\end{abstract}

Keywords: Pigment epithelium detachment, Optical coherence tomography, Choroidal neovascularization, Polypoidal choroidal vasculopathy, Central serous chorioretinopathy, Adult-onset foveal dystrophy.

Accepted on January 04, 2019

\section{Introduction}

The PED and subretinal fluid (SRF) detachment are prominent lesions occurring in many chorioretinal disease processes. The most prevalent aetiology is age-related macular degeneration (AMD). The PED is characterized by the presence of fluid accumulation beneath the retinal pigment epithelium [1] and presents a complex variety of patterns. In the normal eye, photoreceptors are always in contact with the retinal epithelium. The SRF accumulates between the retinal pigment epithelium and the neurosensory retina. Both signs are hyporeflective in a structural OCT. A fusiform, highly-organized, hyper-reflective band could be associated in subtypes of PED (Figure 1) [2-4]. On structural OCT, we can easily identify hypo-reflective fluid accumulation (SRF detachment, intra retinal fluid, PED) and hyper-reflective accumulation (hyperreflective dots), subretinal hyperreflective exudation (SHE), changes in the ellipsoid zone (EZ), external limitant membrane (ELM) and the nuclear layer, suggesting pigment epithelial cell migration [5]. But OCT alone is often not enough to conclude to a diagnosis. To explore the possible etiologies of PED and SRF detachment, some other examinations should also be performed. Fluorescein angiography (FA) and indocyanine green angiography (ICGA) are the gold standards for the multimodal imaging of a retinal-choroidal disease [6-8]. These two exams may be useful for the detection of the aetiology for the PED (retinal/choroidal vascular, tissular changes and disc changes). Additionally, OCT angiography will help evidence the vascular blood flow [9]. Some false interpretations may occur due to the size of the PED (blockage or attenuation of hyper-signal). This case report describes a discussion about etiology of PED in OCT.

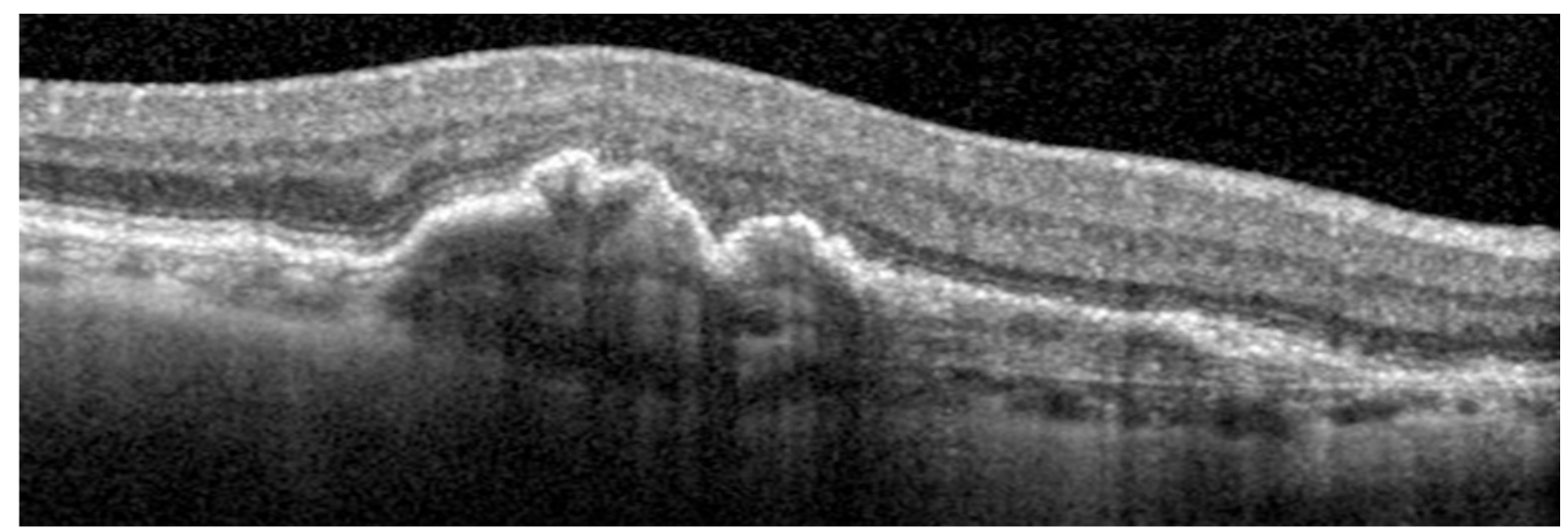

Figure 1: Pigment epithelium detachment without sub retinal fluid.

\section{Case Presentation}

A 70-year-old male patient at the University Eye Clinic of Creteil, France, complained of metamorphopsia and vision loss in his left eye. He was never examined before this consultation in our clinic, had a history of phacoemulsification. Several tests were performed to diagnose him: best corrected visual acuity, bio microscopy, infrared reflectance, auto fluorescence and spectral-domain optical coherence tomography (OCT) 
Citation: Rolf R, Coscas F, Coscas G, Cohen SY, Souied EH. Management of pigment epithelium detachment using Spectral Domain Optical Coherence Tomography. J Clin Ophthalmol 2019;3(1):121-124.

(Spectralis, HRA, Heidelberg, Germany). The clinical examination identified a pigment epithelium detachment (PED), with a visual acuity of 20/100 (Figure 2). This figure shows PED with SRF and intra retinal fluid. The patient presents a PED including an area of irregular and thickened retinal pigment epithelium (RPE) and a heterogenous cavity. It is called fibrovascular PED. It seems to be "occult" choroidal neovascularization (type $1 \mathrm{CNV}$ ) in AMD (Figure 3). We performed anti-vascular endothelial grown factor injection on his left eye.

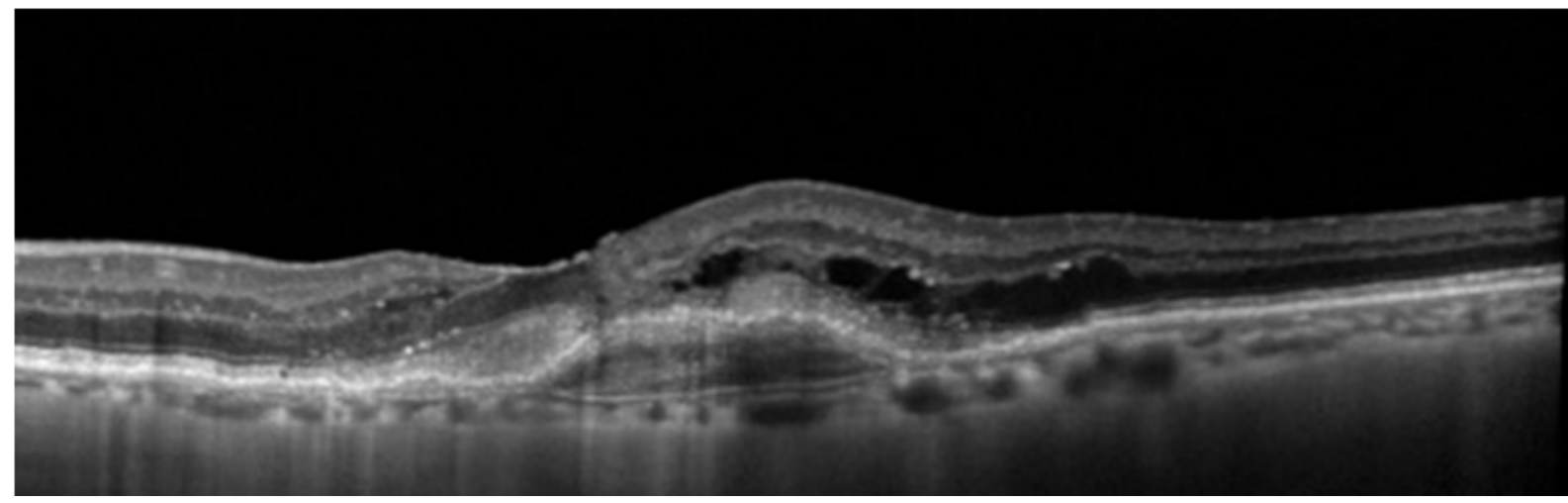

Figure 2: Type I choroidal neo vascularization
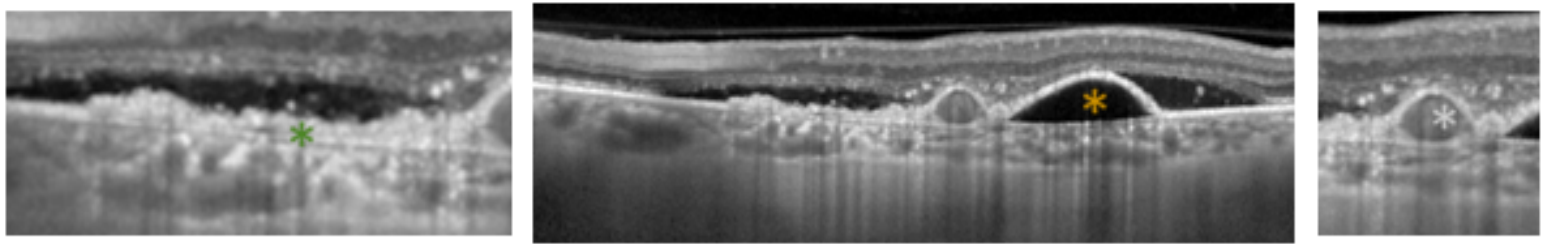

Figure 3: Polypoidal choroidal vasculopathy; the Pigment epithelium detachement (yellow*); posterior surface of the elevated retinal pigment epithelium line. (green*); the branching vascular network (blue*).

\section{Discussion}

The goal of this study is to reinforce the management of pigment epithelium detachment using OCT. Performing OCT to each patient with metamorphopsia, lots of diagnosis are conceivable, and it will be only a good analyze of the images of OCT which allowed us to move forward to the diagnosis.

The patient presents a possible type $1 \mathrm{CNV}$ with fibro vascular PED. The characteristics of PED in type $1 \mathrm{CNV}$ in AMD could be fibro vascular or serous [10]. The serous PED is defined as an area of smooth, sharply demarcated, dome-shaped and regular RPE elevation. The OCT will also show that the cavity remains optically empty [11]. Fluid accumulation remains limited in Type I CNV, versus being accentuated and associated to RPE effraction in Type II and III CNV. The other signs in the OCT, which suggest an occult CNV, are as follows: thin choroid with a few small, irregular, choroidal vessels and rarefied intervascular tissue; some damage in the ellipsoid zone, external limiting membrane and nuclear layer; presence of druse and/or atrophy $[12,13]$.
But it is also very important to know the other possible diagnosis to be able to eliminate them. The retinal specialist needs to analyze the PED, the choroid, the retinal pigment epithelium and all the other layer of the retina.

The PED could have different shape. For example in a polypoidal choroidal vasculopathy (figure 4), the PED (yellow*) is usually hypo-reflective, with a dome-shaped or bumpy well-delineated morphology in which the fluid is trapped, between the RPE and Bruch's membrane [14]. The bump in the RPE line is markedly abrupt [15]. The sub-RPE cavity, with low to medium reflectivity in the central and hyper-reflective borders, looks like the vascular cavity of a polyp. This structure is in close contact with the posterior surface of the elevated RPE line (green*). The branching vascular network (blue*) corresponds to the two hyperreflective lines, representing the retinal pigment epithelium and Bruch membranes, also represented as the so-called "doublelayer" sign. 


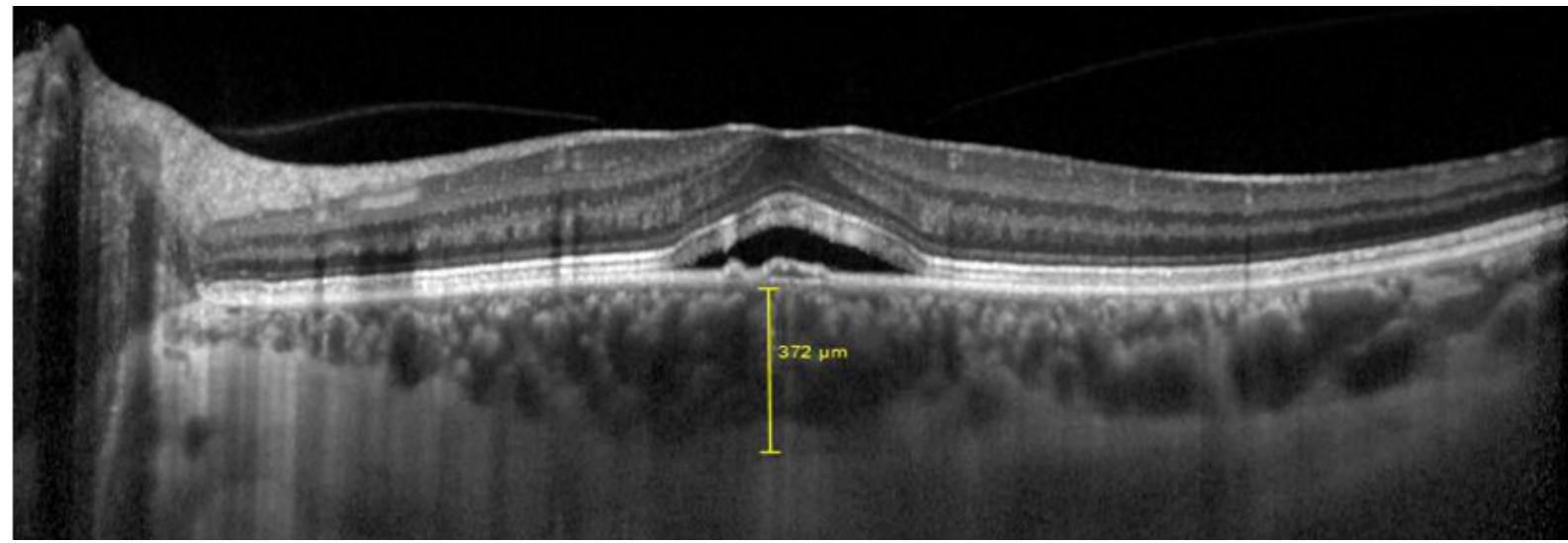

Figure 4: Serous Chrorioretinopathy

Sometimes the serous retinal detachment is relatively flat and larger than the PED. In fact, in a serous chorioretinopathy lesion (CSC) (Figure 5), the PED is only small, and the major sign is not the PED but the serous retinal detachment with limited RPE detachment [16].

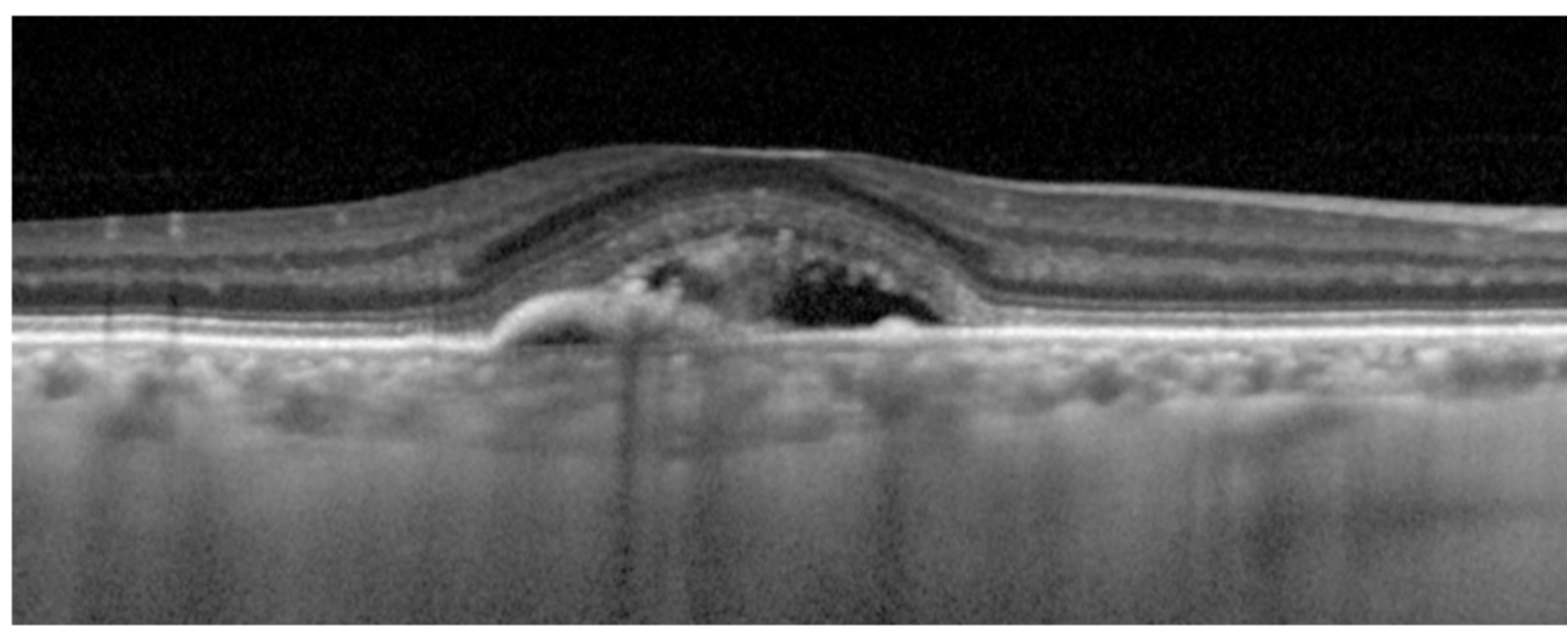

Figure 5: Adult onset foveomacular vitelliform dystrophy (AOFMD).

Between the PED and the SRF, it is interesting to observe the subretinal space above the RPE. Accumlulation of material is a possibility like in adult onset foveomacular vitelliform dystrophy (AOFMD) [17,18].

Finally, the choroid is always very useful to analyse. On the one hand the choroid could be thickened or pachychoroid with enlarged vessels in CSC [19] or in adult onset foveomacular vitelliform dystrophy (Figure 6) [20,21], and on the other hand Thin choroid is described in type $1 \mathrm{CNV}$, with a few small, irregular, choroidal vessels and rarefied intervascular tissue.

In the era of anti-vascular endothelial grown factor (VEGF) therapy, analysing the PED subtype has become more important in helping and identifying its etiology for an indication of the treatment. Anti-VEGF therapy has become the standard of care for neovascular AMD, largely replacing the other options such as verteporfin photodynamic therapy (PDT). Eyes with PCV can be initially treated with anti-VEGF drugs [18]. In the case of acute CSC, the need for treatment depends on the duration and the visual function. There is no effective therapy for AOFMD. Only rehabilitation of the lost vision is an alternative treatment [22].

\section{Conclusion}

Correct diagnosis using OCT allowed the patient to have the appropriate treatment and the good diagnosis. In the era of angio-OCT, semiology is a real challenge. This study shows the importance of basic notion to move in the right direction.

\section{References}

1. Zayit-Soudry S, Moroz I, Loewenstein A. Retinal pigment epithelial detachment. Surv Ophthalmol. 2007;52(3):22743.

2. Rahimy E, Freund KB, Larsen M, Spaide RF, Costa RA, Hoang Q, et al. Multilayered pigment epithelial detachment in neovascular age-related macular degeneration. Retina Phila Pa. 2014;34(7):1289-95.

3. Kadasi LM, Adhi M, Liang MC, et al. Retinal pigment epithelial detachment with disgorgement in age-related macular degeneration observed with OCT. Ophthalmic Surg Lasers Imaging Retina. 2013;44(6 Suppl):S30-32.

4. Lee SY, Stetson PF, Ruiz-Garcia H, et al. Automated characterization of pigment epithelial detachment by 
Citation: Rolf R, Coscas F, Coscas G, Cohen SY, Souied EH. Management of pigment epithelium detachment using Spectral Domain Optical Coherence Tomography. J Clin Ophthalmol 2019;3(1):121-124.

optical coherence tomography. Invest Ophthalmol Vis Sci. 2012;53(1):164-70.

5. Turgut B, Yildirim H. The Causes of Hyperreflective Dots in Optical Coherence Tomography Excluding Diabetic Macular Edema and Retinal Venous Occlusion§. Open Ophthalmol J. 2015;31(9):36-40.

6. Casswell AG, Kohen D, Bird AC. Retinal pigment epithelial detachments in the elderly: classification and outcome. Br J Ophthalmol. 1985;69(6):397-403.

7. Forte R, Querques G, Querques L, et al. Multimodal imaging of dry age-related macular degeneration. Acta Ophthalmol (Copenh). 2012;90(4):e281-7.

8. Querques G, Querques L, Bandello F. Atlas OCT: angiography in AMD: comparison with multimodal imaging. Eur J Ophthalmol. 2015;25(6):e131.

9. Herbort CP, LeHoang P, Guex-Crosier Y. Schematic interpretation of indocyanine green angiography in posterior uveitis using a standard angiographic protocol. Ophthalmology. 1998;105(3):432-40.

10. Kuhn D, Meunier I, Soubrane G, et al. Imaging of chorioretinal anastomoses in vascularized retinal pigment epithelium detachments. Arch Ophthalmol Chic Ill 1960. 1995;113(11):1392-8.

11. Puliafito CA, Hee MR, Lin CP, et al. Imaging of macular diseases with optical coherence tomography. Ophthalmology. 1995;102(2):217-29.

12. Sadda SR, Liakopoulos S, Keane PA, et al. Relationship between angiographic and optical coherence tomographic (OCT) parameters for quantifying choroidal neovascular lesions. Graefes Arch Clin Exp Ophthalmol. 2010 Feb; 248(2):175-84.

13. Hariri A, Heussen FM, Nittala MG, et al. Optical coherence tomographic correlates of angiographic subtypes of occult choroidal neovascularization. Invest Ophthalmol Vis Sci. 2013;54(13):8020-6.

14. Okubo A, Sameshima M, Uemura A, et al. Clinicopathological correlation of polypoidal choroidal vasculopathy revealed by ultrastructural study. $\mathrm{Br} \mathrm{J}$ Ophthalmol. 2002;86(10):1093-8.

15. Alshahrani ST, Al Shamsi HN, Kahtani ES, et al. Spectraldomain optical coherence tomography findings in polypoidal choroidal vasculopathy suggest a type 1 neovascular growth pattern. Clin Ophthalmol Auckl NZ. 2014;8:1689-95.
16. Song IS, Shin YU, Lee BR. Time-periodic characteristics in the morphology of idiopathic central serous chorioretinopathy evaluated by volume scan using spectral-domain optical coherence tomography. Am J Ophthalmol. 2012;154(2):366-375.e4.

17. Kon Y, Iida T, Maruko I, et al. The optical coherence tomography-ophthalmoscope for examination of central serous chorioretinopathy with precipitates. Retina Phila Pa. 2008;28(6):864-9.

18. Gomi F, Oshima Y, Mori R, et al. Initial Versus Delayed Photodynamic Therapy In Combination With Ranibizumab For Treatment Of Polypoidal Choroidal Vasculopathy: The Fujisan Study. Retina Phila Pa. 2015;35(8):1569-76.

19. Nicholson B, Noble J, Forooghian F, Meyerle C. Central serous chorioretinopathy: update on pathophysiology and treatment. Surv Ophthalmol. 2013;58(2):103-26.

20. Gutiérrez-Hernández J-C, Martínez-Camarillo JC, Sadda SR. Long-term follow-up of photoreceptor loss and recovery after half-fluence photodynamic therapy for chronic central serous chorioretinopathy. Retin Cases Brief Rep. 2015;9(2):109-13.

21. Abrishami M, Mousavi M, Hosseini S-M, et al. A. Treatment of Chronic Central Serous Chorioretinopathy with Oral Methotrexate. J Ocul Pharmacol Ther Off J Assoc Ocul Pharmacol Ther. 2015;31(8):468-75.

22. Coscas F, Puche N, Coscas G, et al. Comparison of macular choroidal thickness in adult onset foveomacular vitelliform dystrophy and age-related macular degeneration. Invest Ophthalmol Vis Sci. 2014;55(1):649 .

\section{*Correspondence to}

Rébecca Rolf

University Paris Est Créteil

Centre Hospitalier Intercommunal de Créteil

32 rue Lamarck

75018 PARIS, France

Tel: 0033666987778

E-mail: rebecca.rolf.med@gmail.com 